How to cite this paper:

Shambour, M., K. (2018).Vibrant search mechanism for numerical optimization functions. Journal of Information and Communication Technology. 17(4), 679-702.

\title{
VIBRANT SEARCH MECHANISM FOR NUMERICAL OPTIMIZATION FUNCTIONS
}

\author{
Moh'd Khaled Yousef Shambour \\ Institute for Hajj and Umrah Research \\ Umm Al-Qura University, Saudi Arabia
}

myshambour@uqu.edu.sa

\begin{abstract}
Recently, various variants of evolutionary algorithms have been offered to optimize the exploration and exploitation abilities of the search mechanism. Some of these variants still suffer from slow convergence rates around the optimal solution. In this paper, a novel heuristic technique is introduced to enhance the search capabilities of an algorithm, focusing on certain search spaces during evolution process. Then, employing a heuristic search mechanism that scans an entire space before determining the desired segment of that search space. The proposed method randomly updates the desired segment by monitoring the algorithm search performance levels on different search space divisions. The effectiveness of the proposed technique is assessed through harmony search algorithm (HSA). The performance of this mechanism is examined with several types of benchmark optimization functions, and the results are compared with those of the classic version and two variants of HSA. The experimental results demonstrate that the proposed technique achieves the lowest values (best results) in $80 \%$ of the non-shifted functions, whereas only $33.3 \%$ of total experimental cases are achieved within the shifted functions in a total of 30 problem dimensions. In 100 problem dimensions, $100 \%$ and $25 \%$ of the best results are reported for non-shifted and shifted functions, respectively.
\end{abstract}


The results reveal that the proposed technique is able to orient the search mechanism toward desired segments of search space, which therefore significantly improves the overall search performance of HSA, especially for non-shifted optimization functions.

Keywords: evolutionary algorithms, exploration and exploitation, harmony search algorithm, heuristic search, optimization functions.

\section{INTRODUCTION}

Evolutionary algorithms (EAs) are stochastic optimization algorithms that mimic natural evolution mechanisms, such as mutations and crossovers to solve various optimization problems. EAs have been proven to be effective in various optimization fields due to their efficiency in solving different forms of optimization problems. However, EAs are generally converged to a region after a number of algorithm iterations, but the global optima in this region may not be identified (Yong \& Sannomiya, 2000). Meanwhile, these algorithms gradually lose the diversity among current solutions as iteration counts increase, and thus they prematurely converge into a local optimum. A higher diversity among current solutions is generally important in reducing the chances of premature convergence (Sultan et al., 2004).

Determining the conditions wherein the exploration and exploitation search strategies of an algorithm are balanced is a major challenge encountered in research on EA behavior enhancement. Algorithm performance improves when the balance of the usage is enhanced. Many improved versions of classical EA methods are partly based on this fact and can thus improve algorithm performance in terms of finding global optima, such as genetic algorithm (Holland, 1975), particle swarm optimization (Kennedy and Eberhart, 1995) and harmony search algorithm (Geem et al., 2001).

This paper presents a novel heuristic search technique for solving numerical benchmark functions in which a harmony search algorithm (HSA) is used as a type of EA. HSA is selected because of its general applicability and capability to obtain satisfactory solutions on a wide range of optimization fields (Cobos et al., 2010; Alia \& Mandava, 2011; Lenin et al., 2013; Shambour et al., 2014). The main objective of this study is to control the algorithm search mechanism toward the promising regions of a problem search space and orient the algorithm such that it searches an optimal solution within a reasonable computational time. 
The proposed method launches the process by dividing the search space of a given problem into a random number of equally split segments. Then, it generates several initial solutions for every search segment to identify the best initial solution to be improved. Thereafter, the proposed technique periodically generates new solutions from other segments during algorithm's search process and compared their fitness results with the fitness of current selected segment. The segment with the best solution quality will be only considered for next generation process.

The effectiveness of the proposed technique is demonstrated by using a set of well-known benchmark functions introduced for CEC2005 (Suganthan et al., 2005), and the experimental results are compared with basic HSA and two other variants of HSA.

\section{HARMONY SEARCH ALGORITHM}

Harmony Search Algorithm (HSA) is a heuristic algorithm inspired by the improvisation process of musicians when they compose a well-sounding harmony with their musical instruments. It is considered as a robust algorithm owing to its general applicability to many problems in various optimization fields such as, water distribution networks (Geem, 2009), design of steel structure (Carbas \& Aydogdu, 2007), timetabling problem (Shambour et al., 2013) power dispatch (Valipour \& Ghasemi, 2017), vehicle routing (Yassen et al., 2015), robot application (Xu et al., 2010), design of satellite heat pipe (Geem, 2016), design of aircraft panels (Keshtegar et al., 2017), energyefficient routing network (Zeng \& Dong, 2016) and data Mining applications (Assad \& Deep, 2016).

HSA is characterized by a good exploration of the search space and involves the composition of new solutions from several existing solutions in every algorithm's iteration. However, similar to other optimization algorithms, the HSA is insufficiently capable of generating optimal solutions during the search process (Kazmi et al., 2017).

The main HSA operators include; harmony memory (HM) that reflects population memory which stores several harmony vectors according to the harmony memory size (HMS); and harmony memory consideration rate (HMCR), which denotes the rate of selecting a value with a (i) memory consideration rule, where the decision variable value is extracted from stored harmony vectors in HM, or (ii) memory consideration rule, where the value of decision variable is randomly generated. HSA operators also comprise the pitch adjustment rate (PAR) that indicates the rate of modifying the selected 
value in memory consideration rule to a neighboring value, in addition to bandwidth (BW), which is considered as an adjustment value used in the pitch adjustment rule.

The principal steps of the HSA are explained below and illustrated by a flowchart (Figure 1):

1. Initializing the HSA parameters

This step involves two main sections. The first section includes the initialization of the optimization parameter, HMCR, PAR, BW, and number of iterations (NI). The second section defines the initialization of the problem parameters targeted for resolution.

2. Initializing the HM

HM is initialized with several initial harmony vectors before the start of the algorithm process.

3. Improvisation process

This step is the crucial part of the entire process of the HSA. A new harmony is generated through the use of various HSA operators.

4. Updating the HM

The HM is revised after improvisation by replacing the weakest harmony located with the most promising solution obtained through the improvisation process. The worst harmony vector in the HM has the lowest fitness value among the vectors.

5. Check the stopping criterion

Whether further improvisation required is determined in this final step on the basis of the number of iterations. 


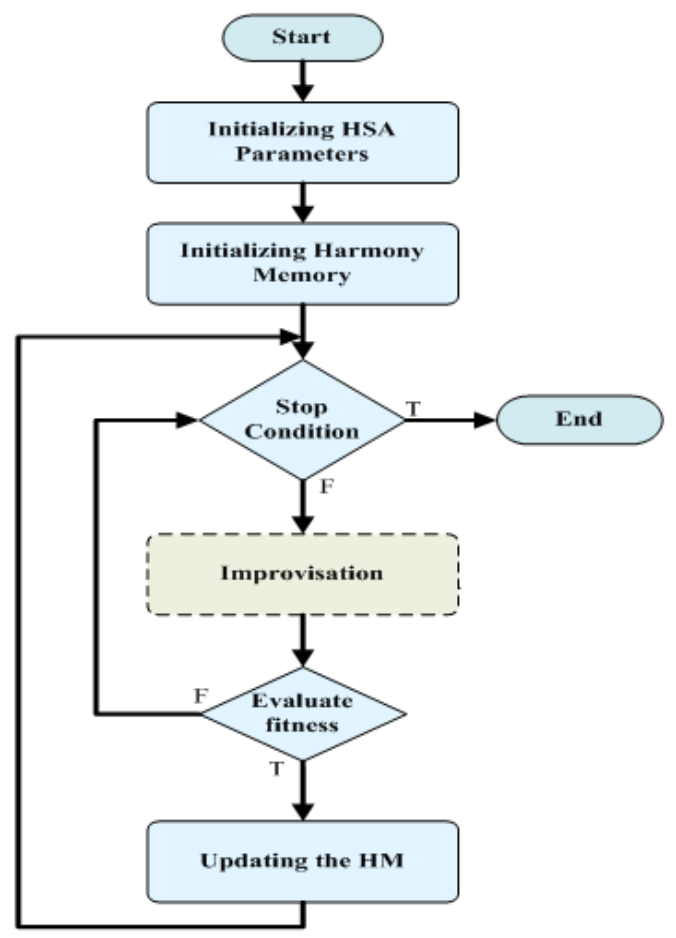

Figure1. HSA Flowchart.

\section{RELATED WORK}

Since the formulation of the first HSA, a series of ameliorated variants of the classic HSA progressed. However, most of the improved versions focus first on improving the algorithm according to three fundamental aspects, namely, the tuning of the optimization parameters, hybridization of the HSA with other parts of other algorithms, and the addition of optimization parameters as criteria for the selection of harmony values from the HM (Shambour et al., 2014).

Early modifications on the HSA parameters were launched by Mahdavi et al. (2007), where the optimization parameter values were dynamically adjusted during improvisation process. They were able to diminish the BW exponentially from the maximum value to the minimum value but facilitate the linear growth of PAR values from the minimum value to the maximum value. 
Contreras et al. (2014) suggested a new type of HSA named ABHS. This HSA variant consists of the linear augmentation of the PAR value throughout the algorithm iteration processes. However, the BW value in this variant increases constantly and decreases exponentially periodically according to the number of harmonies that penetrate the HM.

Ouyang et al. (2017) proposed a different improved version of HSA with integrated principal features, namely, adaptive global pitch adjustment, opposition-based learning technique, and mechanism selection. This technique adjusts the BW parameter according to the status of the best and worst harmony vectors in HM to enhance the algorithm exploitation power of the search space.

Another modification of HSA was introduced by Kumar et al. (2014). In this HAS version, improvisation starts with a minor HMCR value, which increases linearly during algorithm iterations. This increase facilitates investigation within the search space. The PAR value is reduced exponentially for the improvement of the solutions.

Khalili et al. (2014) proposed a free predefined parameter setting of HSA. In this setting, HMCR, PAR, and BW parameters are dynamically changed according to the nature of a targeted problem. At the early improvisation stage, the algorithm starts elaborating the search solution by generating harmonies rather than selecting solutions from actual HM. Meanwhile, low PAR values can be adjusted or repeated depending on the proposed search mechanism. At the middle stage of the improvisation process, large HMCR values are increased to 1 for the selection of decision variable values from HM. PAR values are then increased to 1 for the adjustment procedure. At subsequent iterations, the HMCR value declines and subsequently enables the algorithm to escape from local minima by forcing a random consideration procedure instead of a memory consideration. Further related research can be found in (Sabarinath et al., 2015; Kong et al., 2009).

Hybridization provides enhanced convergence speed and accuracy for evolutionary approaches (Ibrahim et al., 2015). Omran et al. (2008) are among the pioneers of hybridization techniques for HSA. The main idea of their work is to inject the principal of the particle swarm optimization within the HSA core. The inserted part is in the pitch adjustment rule where the BW parameter is exchanged by an inherited value of the best harmony vector in HM.

Shambour et al. (2013) studied the proposition of a heuristic technique that combines HSA and simulated annealing (SA) method to solve "RealWorld" high school timetabling optimization problem. The SA method is employed at the end of every improvisation process for the enhancement of a generated harmony. Five neighborhood functions, namely move meeting, 
swap meeting, swap three meetings, swap block of meetings, and task split functions are randomly selected and applied to the newly generated harmony.

Satapathy et al. (2017) formulated a hybrid HSA with firefly algorithm (FA) to investigate the stability profile improvement of a distributed resourcebased microgrid. An improved version fruitfully increases population diversity. Meanwhile, FA is generally used for increasing convergence speed.

An improved approach of HSA and Cuckoo-Search (CS) is produced by Wang et al. (2016) to resolve numerical benchmark functions. In this approach, the search capability of CS is enhanced by hybridizing HSA and $\mathrm{CS}$, and the pitch adjustment operator of HSA is included in the CS process, thereby increasing convergence rate during a search process. Hasan et al. (2014) investigated the performance of HSA by exchanging a stochastic operator with crossover processes. For other related work, refer to Zhang et al. (2015), and Kar \& Swain (2016).

Shambour et al. (2014) offered a new method for adjusting the tournament selection scheme of HSA. In this method, the selection procedure is performed before the tournament scheme operation, and a random selection is performed on the basis of stochastic tournament sizes. The most appropriate solution vector selected from different competitive harmonies is utilized for further processing.

In another study, Shambour (2017) enhanced the exploration behavior of the classical HSA through monitoring the search performance on different subzones of the whole search space. Thereafter, the subzone with the best search performance will be considered for further search process. The proposed algorithm is validated on twelve numerical benchmark functions and the results show advantages of the proposed algorithm compared to the previous improved algorithms.

Al-Betar et al. (2012) worked on the impact of various selection schemes of HSA on improvising a new solution. The study is based on exchanging the classic random selection process by a number of other schemes (globalbest, linear rank, tournament, fitness-proportional and exponential rank). Other work studies that improvised traditional HSA through various selection schemes can be found in (Chen et al., 2012; Doush et al., 2013; Al-Betar et al., 2016).

All the previous work mentioned above are focused on the search capabilities of the HSA and designing methods for the enhancement of basic algorithm performance quality in various optimization fields. However, some room remains for ameliorating the efficiency of the HSA through controlling the exploration process of the random search mechanism. This can be achieved by guiding the search progress in exploring certain promising areas of the search space during the algorithm search progress. 


\section{PROPOSED METHOD}

The random variation part of optimization algorithms continues to attract interest among researchers of the evolution search process. These compromise trials enrich the generation by exploring more areas of the search space through supporting a series of random variables.

VSM relies on promoting certain changes to the basic HSA. Figure 2 shows the modifications of the original HSA which can be explained as follows:

\section{Division Stage}

In this stage, the search space is first split into random divisions (up to ten). Each division has the same size and probability of selection. The division of the search space enables direct searching.

\section{Production Stage}

This stage involves the production of initial solutions according to the number of previously created divisions. The lowest average of the values of initial solutions in each subdivision is used for the identification of a promising subdivision.

\section{Orientation Stage}

In the third stage, the algorithm search mechanism orients the search toward the promising subdivision, and the initial solutions of the selected subdivision is inserted into HM. Then, the improvisation procedure is employed on the promising division for the generation of harmonies that may contain an optimal solution.

\section{Detection Stage}

A detection procedure is required in a periodical mode and must consider all subdivisions produced in step 1 . This procedure is necessary to the validation of the suitability of a selected subdivision. The recurring process will be based on detection rate (DR) that is created randomly and ranges between 0 and 0.01 . That is, $\mathrm{DR}=[0.000,0.0025,0.005,0.0075,0.01]$. This phase promotes a reassuring aspect of previous processes and verifies the presence of any division containing a promising subdivision. This procedure directs the search process toward the promising division, thereby improving the accuracy of results. 
The number of dedicated iteration (DI) to detection procedure is given in equation 1 .

$$
D I=N I \times D R[U(1,5)]
$$

Where NI is the maximum number of iterations, and $U$ is uniform random integer between $(1,5)$. The pseudocode of the proposed algorithm is provided in Figure 3.

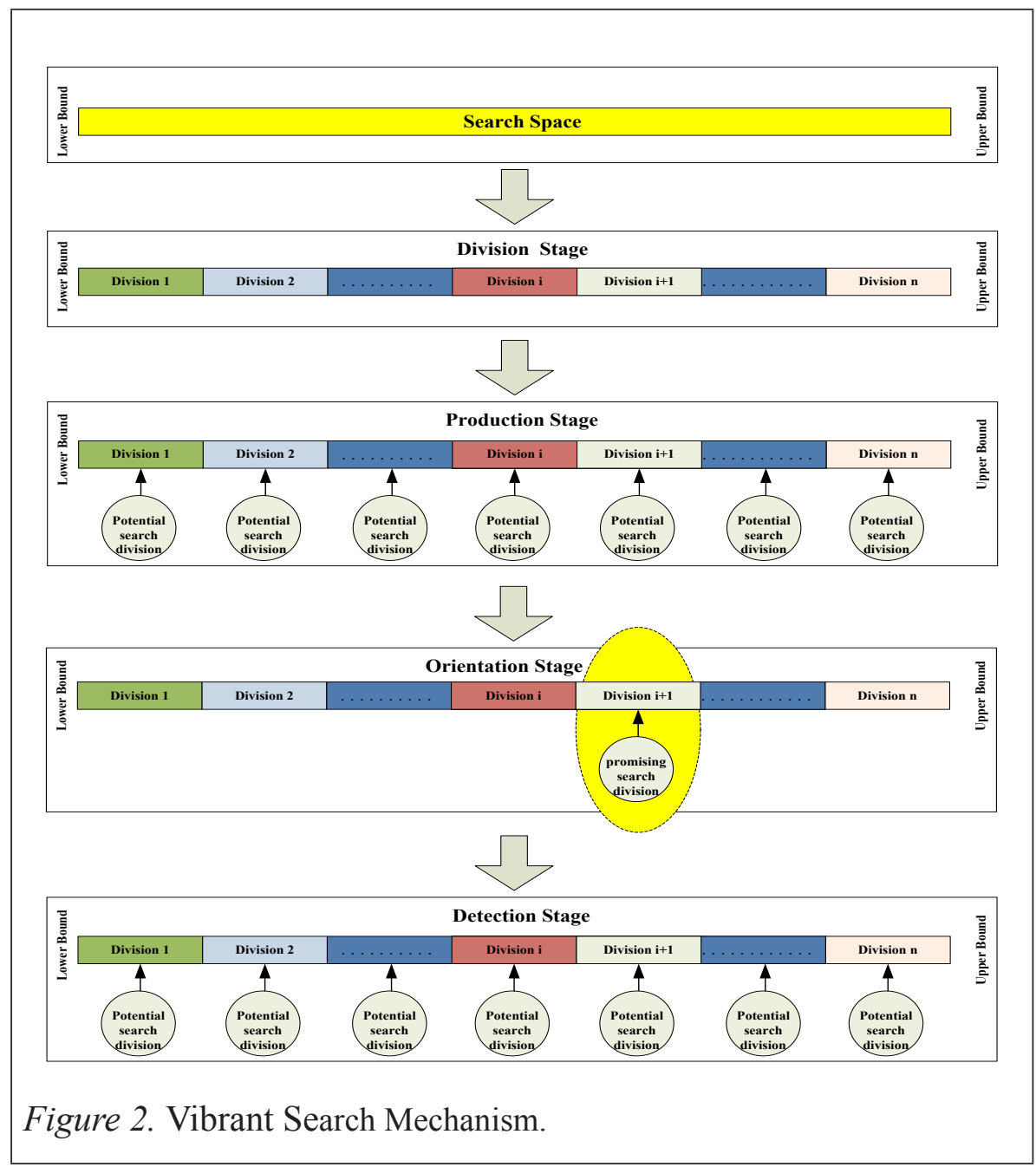




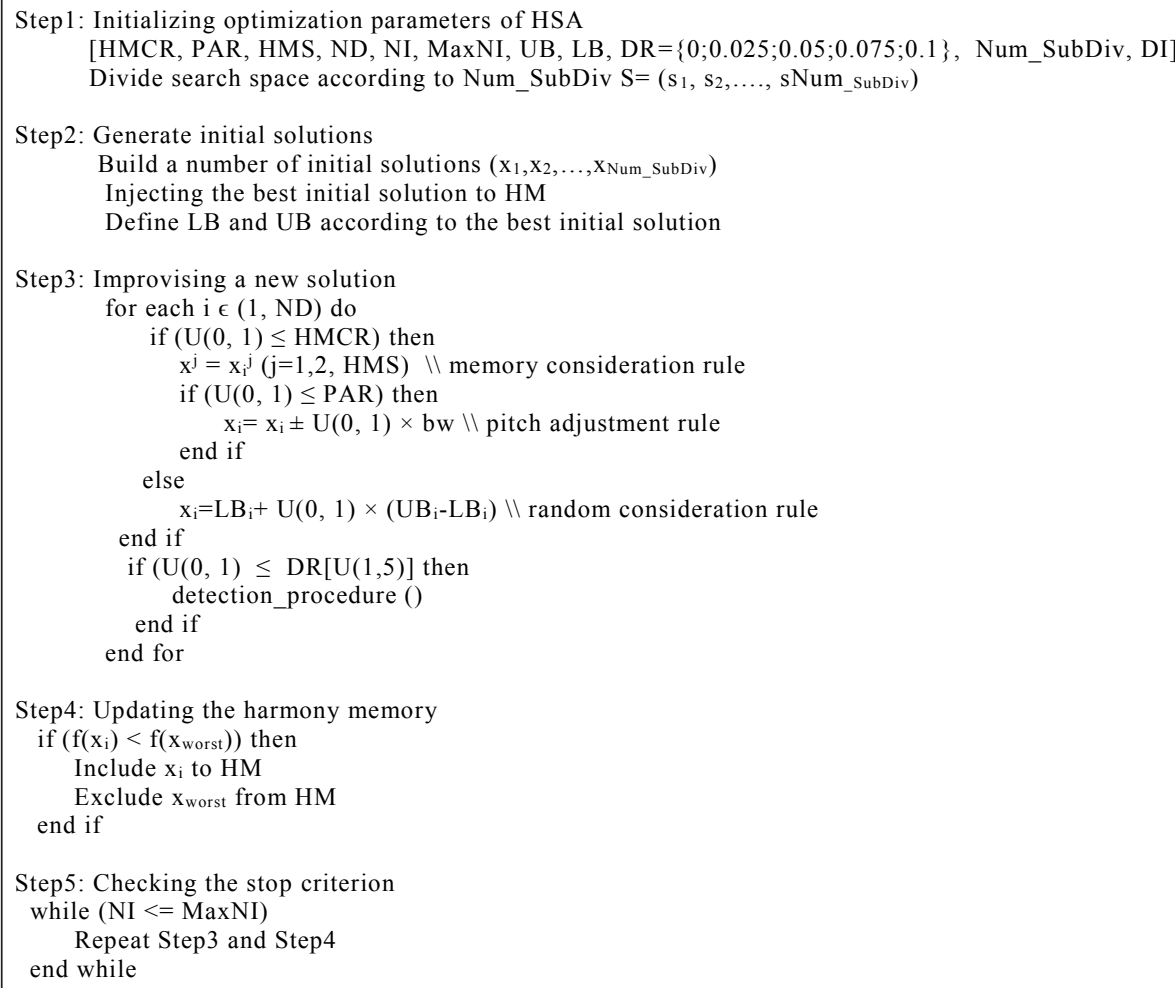

Step5: Checking the stop criterion

while $(\mathrm{NI}<=$ MaxNI)

Repeat Step3 and Step4

end while

Figure 3. The proposed Vibrant Search Mechanism.

\section{EMPIRICAL EXPERIMENTS AND RESULTS}

The performance of the proposed VSM is validated through a series of numerical simulations and compared with three HSA variants namely, original HSA (OHSA) (Geem et al., 2001), tournament HSA (THSA), and Global HSA (GHSA) (Al-Betar et al., 2012) where a group of benchmark optimization functions including different specifications (i.e. uni-modal, multi-modal, continues, and so on) are used to examine the effectiveness and robustness of the proposed mechanism in terms of result accuracy.

\section{Numerical Benchmark Optimization Functions}

A total of 14 well-known numerical benchmark instances introduced for CEC 2005 (Suganthan et al., 2005) are taken in this study. The definitions of numerical instances and their properties are presented in Table 1. The two dimension landscapes of benchmark functions are given in Figure 4. 
Table 1

Benchmark optimization functions

\section{1) Sphere function [Unimodal]}

$f_{1}(x)=\sum_{i=1}^{N} x_{i}^{2}$

$x_{i} \in[-100,100], \quad \min \left(f_{1}\right)=f(0, \ldots, 0)=0$

2 )Scwefel's problem 2.22 [Unimodal]

$$
\begin{aligned}
& f_{2}(x)=\sum_{i=1}^{D}\left|x_{i}\right|+\prod_{i=1}^{D}\left|x_{i}\right| \\
& x_{i} \in[-10,10], \min \left(f_{2}\right)=f(0, \ldots, 0)=0-
\end{aligned}
$$

3)Step function/Unimodal \& discontinues]

$$
\begin{aligned}
& f_{3}(x)=\sum_{i=1}^{N} \mid\left(\left\lfloor x_{i}+0.5\right\rfloor\right)^{2} \\
& x_{i} \in[-100,100], \quad \min \left(f_{3}\right)=f(0, \ldots, 0)=0
\end{aligned}
$$

4) Rosenbrock function [Multimodal]

$$
\begin{aligned}
& f_{4}(x)=\sum_{i=1}^{D-1}\left(100\left(x_{i}^{2}-x_{i+1}\right)^{2}+\left(x_{i}-1\right)^{2}\right) \\
& x_{i} \in[-30,30], \quad \min \left(f_{4}\right)=f(1, \ldots, 1)=0
\end{aligned}
$$

\section{8) Ackley's function [Multimodal]}

$f_{8}(x)=-20 \exp \left(-0.2 \sqrt{\frac{1}{D} \sum_{i=1}^{D} x_{i}^{2}}\right)-\exp \left(\frac{1}{D} \sum_{i=1}^{D} \cos \left(2 \pi x_{i}\right)\right)+20+e$

$x_{i} \in[-100,100], \min \left(f_{3}\right)=f(0, \ldots, 0)=0$

9)Griewank function [Multimodal]

$f_{9}(x)=\sum_{i=1}^{D} \frac{x_{i}{ }^{2}}{4000}-\prod_{i=1}^{D} \cos \left(\frac{x_{i}}{\sqrt{i}}\right)+1$

$x_{i} \in[-600,600], \min \left(f_{9}\right)=f(0, \ldots .0)=0$

\section{0) Six-Hump Camel-Back function [Multimodal]}

$f_{10}(x)=4 x_{1}^{2}-2.1 x_{1}^{4}+\frac{1}{3} x_{1}^{6}+x_{1} x_{2}-4 x_{2}^{2}+4 x_{2}^{4}$

$x_{i} \in[-5,5], \quad \min \left(f_{10}\right)=f(0.08983,0.7126)=1.0316285$

\section{1) Shifted Sphere function [Unimodal]}

$f_{11}(x)=\sum_{i=1}^{N} z_{i}^{2}+f_{\text {_ }}$ iias $_{1}, z=x-o$

$x_{i} \in[-100,100], \quad \min \left(f_{11}\right)=\mathrm{f}\left(\mathrm{o}_{1}, \ldots, \mathrm{o}_{\mathrm{N}}\right)=\mathrm{f}_{\text {-bias } 1}=-450$

\section{5) Rotated hyper-ellipsoid function [Unimodal] 12) Shifted Rosenbrock [Multimodal]}

$$
\begin{aligned}
& f_{5}(x)=\sum_{i=1}^{N} \mid\left(\sum_{j=1}^{i} x_{j}\right)^{2} \\
& x_{i} \in[-100,100], \quad \min \left(f_{5}\right)=f(0, \ldots, 0)=0
\end{aligned}
$$

6) Schwefel's problem [Multimodal]

$$
f_{6}(x)=-\sum_{i=1}^{D} x_{i} \sin \left(\left|x_{i}\right|^{1 / 2}\right)
$$

$x_{i} \in[-500,500], \min \left(f_{6}\right)=f(420.9687, \ldots, 420.9687)=-12569.5$

\section{7) Rastrigin function [Multimodal]}

$$
\begin{aligned}
& f_{7}(x)=\sum_{i=1}^{D}\left(x_{i}{ }^{2}-10 \cos \left(2 \pi x_{i}\right)+10\right) \\
& x_{i} \in[-5.12,5.12], \quad \min \left(f_{7}\right)=f(0, \ldots, 0)=0
\end{aligned}
$$

$$
\begin{aligned}
& f_{12}(x)=\sum_{i=1}^{N-1}\left(100\left(z_{i+1}-z_{i}^{2}\right)^{2}+\left(z_{i}-1\right)^{2}\right)+f_{-} \text {bias }_{6}, z=x-o \\
& x_{i} \in[-100,100], \quad \min \left(f_{12}\right)=\mathrm{f}\left(\mathrm{o}_{1}, \ldots, \mathrm{o}_{\mathrm{N}}\right)=\mathrm{f}_{\text {-bias } 6}=-390
\end{aligned}
$$

\section{3) Shifted Rosenbrock [Multimodal]}

$$
f_{13}(x)=\sum_{i=1}^{N-1}\left(100\left(z_{i+1}-z_{i}^{2}\right)^{2}+\left(z_{i}-1\right)^{2}\right)+f \text { bias }_{6}, z=x-o
$$$$
x_{i} \in[-100,100], \quad \min \left(f_{13}\right)=\mathrm{f}\left(\mathrm{o}_{1}, \ldots, \mathrm{o}_{\mathrm{N}}\right)=\mathrm{f}_{\text {-bias } 6}=-390
$$

\section{4) Shifted Rastrigin [Multimodal]}

$$
\begin{aligned}
& f_{14}(x)=\sum_{i=1}^{N}\left(z_{i}^{2}-10 \cos \left(2 \pi z_{i}\right)+10\right)+f_{-} \text {bias }_{9}, z=x-o \\
& x_{i} \in[-5,5], \quad \min \left(f_{14}\right)=\mathrm{f}\left(\mathrm{o}_{1}, \ldots, \mathrm{o}_{\mathrm{N}}\right)=\mathrm{f}_{\text {-bias }}=-330
\end{aligned}
$$

\section{Experimental Setup}

Similar values of optimization parameters are set in the initialization step for all compared HSA variants (i.e. OHSA, THSA and GHSA), where HMCR $=0.95$, $\mathrm{PAR}=0.3$, number of iterations $N I=20000, D R=[0 ; 0.025 ; 0.05 ; 0.075 ; 0.1]$, $D I=N I \times D R[U(1,5)]$, maximum number of sub divisions Max_SubDiv=10, the dimensionality $N D=30$, with different values of $\operatorname{HMS}$ including $(5,20$ and 50). The proposed mechanism is coded using Matlab simulation program version 2012b and performed on a computer with Microsoft Windows 7 (64bit) with Intel processor i5@3.4 GHz. 


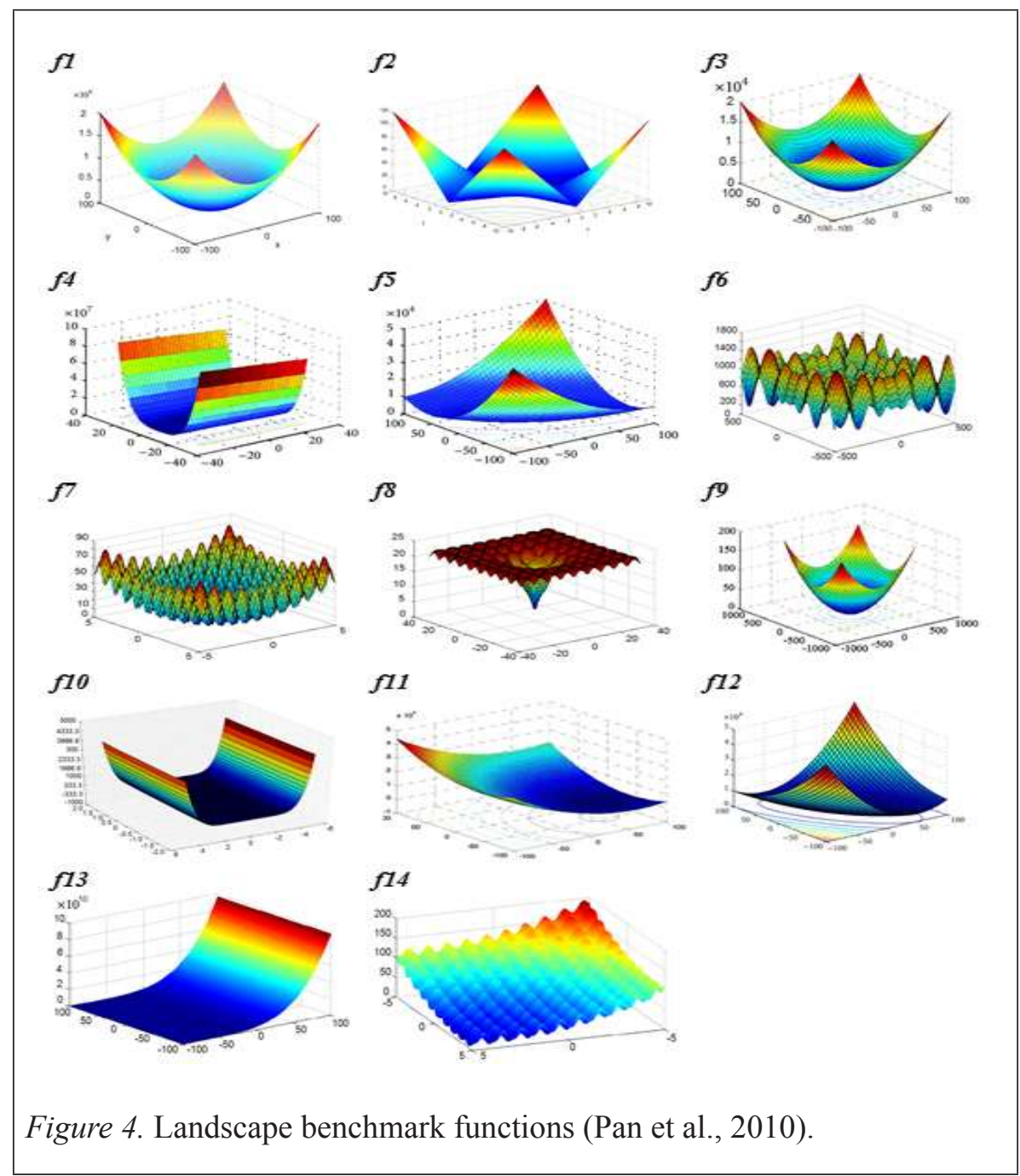

\section{Results and discussion}

The detection procedure of VSM randomly scans the entire search space, including the created subdivisions, to define the promising division that requires further investigation. This scanning process is performed once at the start of the improvisation stage and periodically in a random manner during the process according to a predefined $D R$.

The experiments tackle different HMSs and consider the value of maximum number of subdivisions randomly set between 1 and 10 in each run.

Tables 2, 3, and 4 show the statistical results in terms of the solution fitness value, including best, mean, and standard deviation for 30 runs for 
each compared HSA variant (i.e. OHSA, GHSA, THSA, and VSM) with 30 problem dimensions. The bold font highlights the lowest solutions which reflect the best results, as this study deals with minimizing problems.

Table 2

Best, Mean and Standard Deviation of the Benchmark Function Results $(H M S=5, N D=30)$

\begin{tabular}{|c|c|c|c|c|c|}
\hline $\begin{array}{l}\text { Benchmark } \\
\text { function }\end{array}$ & & $O H S A$ & $G H S A$ & $T H S A$ & $V S M$ \\
\hline & Best & $6.536 \mathrm{E}-01$ & $2.523 \mathrm{E}-03$ & $1.13 \mathrm{E}+00$ & $1.435 E-04$ \\
\hline \multirow[t]{2}{*}{$f 1$} & Mean & $5.231 \mathrm{E}+00$ & $2.114 \mathrm{E}+00$ & $3.07 \mathrm{E}+00$ & $2.957 \mathrm{E}+00$ \\
\hline & Std. & $2.887 \mathrm{E}+00$ & $1.936 \mathrm{E}+00$ & $1.48 \mathrm{E}+00$ & $2.856 \mathrm{E}+00$ \\
\hline \multirow{3}{*}{$f 2$} & Best & $4.143 \mathrm{E}-02$ & $3.948 \mathrm{E}-02$ & $3.21 \mathrm{E}-02$ & $3.769 \mathrm{E}-02$ \\
\hline & Mean & 7.079E-02 & 7.055E-02 & $5.43 \mathrm{E}-02$ & $6.021 \mathrm{E}-02$ \\
\hline & Std. & $4.108 \mathrm{E}-02$ & 4.379E-02 & $2.77 \mathrm{E}-02$ & $3.238 \mathrm{E}-02$ \\
\hline \multirow{3}{*}{$f 3$} & Best & $0.000 E+00$ & $1.000 \mathrm{E}+00$ & $1.00 \mathrm{E}+00$ & $0.000 E+00$ \\
\hline & Mean & $4.300 \mathrm{E}+00$ & $3.967 \mathrm{E}+00$ & $4.17 \mathrm{E}+00$ & $3.833 \mathrm{E}+00$ \\
\hline & Std. & $2.395 \mathrm{E}+00$ & $3.011 \mathrm{E}+00$ & $2.77 \mathrm{E}+00$ & $5.596 \mathrm{E}+00$ \\
\hline \multirow{3}{*}{ f4 } & Best & $7.593 \mathrm{E}+01$ & $6.408 \mathrm{E}+00$ & $2.11 \mathrm{E}+01$ & $2.841 \mathrm{E}+00$ \\
\hline & Mean & $4.714 \mathrm{E}+02$ & $3.594 \mathrm{E}+02$ & $2.49 \mathrm{E}+02$ & $1.197 \mathrm{E}+02$ \\
\hline & Std. & $7.195 \mathrm{E}+02$ & $6.871 \mathrm{E}+02$ & $4.85 \mathrm{E}+02$ & $1.065 \mathrm{E}+02$ \\
\hline \multirow{3}{*}{$f 5$} & Best & $5.531 \mathrm{E}+03$ & $2.471 \mathrm{E}+03$ & $4.42 \mathrm{E}+03$ & $1.946 \mathrm{E}-01$ \\
\hline & Mean & $8.876 \mathrm{E}+03$ & $6.867 \mathrm{E}+03$ & $7.99 \mathrm{E}+03$ & $1.211 \mathrm{E}+03$ \\
\hline & Std. & $1.888 \mathrm{E}+03$ & $2.203 \mathrm{E}+03$ & $2.10 \mathrm{E}+03$ & $1.618 \mathrm{E}+03$ \\
\hline \multirow{3}{*}{ f6 } & Best & $-1.26 E+04$ & $-1.26 E+04$ & $-1.26 E+04$ & $-1.26 \mathrm{E}+04$ \\
\hline & Mean & $-1.254 \mathrm{E}+04$ & $-1.254 \mathrm{E}+04$ & $-1.25 \mathrm{E}+04$ & $-1.243 \mathrm{E}+04$ \\
\hline & Std. & $1.589 \mathrm{E}+01$ & $9.695 \mathrm{E}+00$ & $1.22 \mathrm{E}+01$ & $6.460 \mathrm{E}+02$ \\
\hline \multirow{3}{*}{$f 7$} & Best & $2.662 \mathrm{E}-02$ & $3.163 \mathrm{E}-02$ & $2.40 \mathrm{E}-02$ & $1.886 \mathrm{E}-02$ \\
\hline & Mean & $9.532 \mathrm{E}-01$ & $1.034 \mathrm{E}+00$ & $1.19 \mathrm{E}+00$ & $2.072 \mathrm{E}+00$ \\
\hline & Std. & $8.084 \mathrm{E}-01$ & $9.769 \mathrm{E}-01$ & $1.12 \mathrm{E}+00$ & $5.340 \mathrm{E}+00$ \\
\hline \multirow{3}{*}{$f 8$} & Best & $6.744 \mathrm{E}-01$ & $2.337 \mathrm{E}-02$ & $9.88 \mathrm{E}-03$ & $7.885 E-03$ \\
\hline & Mean & $1.287 \mathrm{E}+00$ & $1.120 \mathrm{E}+00$ & $1.02 \mathrm{E}+00$ & $8.949 \mathrm{E}-01$ \\
\hline & Std. & $2.906 \mathrm{E}-01$ & 5.147E-01 & $5.21 \mathrm{E}-01$ & $5.714 \mathrm{E}-01$ \\
\hline \multirow{3}{*}{$f^{9}$} & Best & $1.043 \mathrm{E}+00$ & $1.038 \mathrm{E}+00$ & $1.00 \mathrm{E}+00$ & $8.205 E-02$ \\
\hline & Mean & $1.101 \mathrm{E}+00$ & $1.091 \mathrm{E}+00$ & $1.08 \mathrm{E}+00$ & $1.025 \mathrm{E}+00$ \\
\hline & Std. & $3.515 \mathrm{E}-02$ & $4.168 \mathrm{E}-02$ & $3.08 \mathrm{E}-02$ & $2.459 \mathrm{E}-01$ \\
\hline \multirow{3}{*}{ f10 } & Best & $-1.032 E+00$ & $-1.032 E+00$ & $-1.03 E+00$ & $-1.032 E+00$ \\
\hline & Mean & $-1.032 \mathrm{E}+00$ & $-1.032 \mathrm{E}+00$ & $-1.03 E+00$ & $-1.032 \mathrm{E}+00$ \\
\hline & Std. & $4.728 \mathrm{E}-10$ & $1.461 \mathrm{E}-10$ & $3.04 \mathrm{E}-10$ & $1.908 \mathrm{E}-10$ \\
\hline \multirow{3}{*}{$f 11$} & Best & $1.220 \mathrm{E}+00$ & $2.057 \mathrm{E}-03$ & $2.57 \mathrm{E}-01$ & $1.608 \mathrm{E}+00$ \\
\hline & Mean & $5.508 \mathrm{E}+00$ & $1.450 \mathrm{E}+00$ & $4.19 \mathrm{E}+00$ & $3.944 \mathrm{E}+03$ \\
\hline & Std. & $3.442 \mathrm{E}+00$ & $1.174 \mathrm{E}+00$ & $2.59 \mathrm{E}+00$ & $1.251 \mathrm{E}+04$ \\
\hline \multirow{3}{*}{$f 12$} & Best & $4.638 \mathrm{E}+03$ & $3.170 \mathrm{E}+03$ & $5.11 \mathrm{E}+03$ & $9.621 \mathrm{E}+03$ \\
\hline & Mean & $1.013 \mathrm{E}+04$ & $9.557 \mathrm{E}+03$ & $9.58 \mathrm{E}+03$ & $1.897 \mathrm{E}+04$ \\
\hline & Std. & $3.244 \mathrm{E}+03$ & $3.466 \mathrm{E}+03$ & $3.32 \mathrm{E}+03$ & $3.856 \mathrm{E}+03$ \\
\hline \multirow{3}{*}{$f 13$} & Best & $4.445 \mathrm{E}+02$ & $2.976 \mathrm{E}+02$ & $4.42 \mathrm{E}+03$ & $4.703 \mathrm{E}+02$ \\
\hline & Mean & $3.219 \mathrm{E}+03$ & $1.690 \mathrm{E}+03$ & $7.99 \mathrm{E}+03$ & $2.059 \mathrm{E}+09$ \\
\hline & Std. & $3.004 \mathrm{E}+03$ & $3.546 \mathrm{E}+03$ & $2.10 \mathrm{E}+03$ & $5.185 \mathrm{E}+09$ \\
\hline \multirow{3}{*}{ f14 } & Best & $3.059 \mathrm{E}-02$ & $3.135 \mathrm{E}-02$ & $-1.25 \mathrm{E}+04$ & $2.603 E-02$ \\
\hline & Mean & $1.358 \mathrm{E}+00$ & $8.861 \mathrm{E}-01$ & $1.22 \mathrm{E}+01$ & $3.738 \mathrm{E}+01$ \\
\hline & Std. & $1.218 \mathrm{E}+00$ & $9.022 \mathrm{E}-01$ & $1.13 \mathrm{E}+00$ & $7.106 \mathrm{E}+01$ \\
\hline
\end{tabular}


The observed results generally show that the proposed VSM method has superior performance in most experiment cases of non-shifted functions set (i.e. $f 1-f 10$ ) with $80 \%$ of total experiment cases and acceptable performance with $33.3 \%$ for the shifted set when compared with other HSA variants.

The results demonstrate that the proposed VSM achieves the best results compared with other algorithms in 10 out of 14 experimental cases when the HMS is equal to 5 and 20 and in seven cases when HMS is equal t.

Table 3

Best, Mean and Standard Deviation of the Benchmark Function Results $(H M S=20, N D=30)$

\begin{tabular}{|c|c|c|c|c|c|}
\hline $\begin{array}{l}\text { Benchmark } \\
\text { function }\end{array}$ & & OHSA & $G H S A$ & $T H S A$ & VSM \\
\hline \multirow{3}{*}{$f 1$} & Best & $4.543 \mathrm{E}+00$ & $1.484 \mathrm{E}-02$ & $3.025 \mathrm{E}+00$ & $1.483 E-01$ \\
\hline & Mean & $9.826 \mathrm{E}+00$ & $1.466 \mathrm{E}+00$ & $9.527 \mathrm{E}+00$ & $7.18 \mathrm{E}+00$ \\
\hline & Std. & $3.964 \mathrm{E}+00$ & $1.250 \mathrm{E}+00$ & $4.885 \mathrm{E}+00$ & $4.27 \mathrm{E}+00$ \\
\hline \multirow{3}{*}{$f^{2}$} & Best & $5.195 \mathrm{E}-02$ & $4.089 \mathrm{E}-02$ & $3.965 \mathrm{E}-02$ & 3.903E-02 \\
\hline & Mean & $1.446 \mathrm{E}-01$ & 7.937E-02 & 7.392E-02 & 7.981E-02 \\
\hline & Std. & 8.874E-02 & $3.495 \mathrm{E}-02$ & 3.699E-02 & $3.458 \mathrm{E}-02$ \\
\hline \multirow{3}{*}{$f 3$} & Best & $1.000 \mathrm{E}+00$ & $0.000 \mathrm{E}+00$ & $0.000 E+00$ & $0.000 \mathrm{E}+00$ \\
\hline & Mean & $6.400 \mathrm{E}+00$ & $3.800 \mathrm{E}+00$ & $4.433 \mathrm{E}+00$ & $4.133 \mathrm{E}+00$ \\
\hline & Std. & $3.349 \mathrm{E}+00$ & $3.357 \mathrm{E}+00$ & $3.857 \mathrm{E}+00$ & $3.711 \mathrm{E}+00$ \\
\hline \multirow{3}{*}{$f 4$} & Best & $1.104 \mathrm{E}+02$ & $1.321 \mathrm{E}+00$ & $1.071 \mathrm{E}+02$ & $1.768 \mathrm{E}+01$ \\
\hline & Mean & $4.161 \mathrm{E}+02$ & $2.250 \mathrm{E}+02$ & $3.106 \mathrm{E}+02$ & $2.146 \mathrm{E}+02$ \\
\hline & Std. & $3.850 \mathrm{E}+02$ & $4.072 \mathrm{E}+02$ & $4.311 \mathrm{E}+02$ & $1.155 \mathrm{E}+02$ \\
\hline \multirow{3}{*}{$f 5$} & Best & $4.393 \mathrm{E}+03$ & $2.627 \mathrm{E}+03$ & $4.863 \mathrm{E}+03$ & $4.874 E+00$ \\
\hline & Mean & $7.269 \mathrm{E}+03$ & $8.276 \mathrm{E}+03$ & $7.774 \mathrm{E}+03$ & $1.578 \mathrm{E}+03$ \\
\hline & Std. & $2.636 \mathrm{E}+03$ & $2.996 \mathrm{E}+03$ & $2.268 \mathrm{E}+03$ & $2.486 \mathrm{E}+03$ \\
\hline \multirow{3}{*}{ f6 } & Best & $-1.255 \mathrm{E}+04$ & $-1.256 \mathrm{E}+04$ & $-1.255 \mathrm{E}+04$ & $-1.257 E+04$ \\
\hline & Mean & $-1.253 \mathrm{E}+04$ & $-1.254 \mathrm{E}+04$ & $-1.254 \mathrm{E}+04$ & $-1.254 \mathrm{E}+04$ \\
\hline & Std. & $1.224 \mathrm{E}+01$ & $1.020 \mathrm{E}+01$ & $1.067 \mathrm{E}+01$ & $2.067 \mathrm{E}+01$ \\
\hline \multirow{3}{*}{$f 7$} & Best & $6.084 \mathrm{E}-02$ & $3.555 \mathrm{E}-02$ & 3.429E-02 & $2.177 E-02$ \\
\hline & Mean & $1.473 \mathrm{E}+00$ & $1.376 \mathrm{E}+00$ & $1.014 \mathrm{E}+00$ & $1.741 \mathrm{E}+00$ \\
\hline & Std. & $9.824 \mathrm{E}-01$ & $1.163 \mathrm{E}+00$ & $8.710 \mathrm{E}-01$ & $5.384 \mathrm{E}+00$ \\
\hline \multirow{3}{*}{$f 8$} & Best & $6.444 \mathrm{E}-01$ & $1.516 \mathrm{E}-02$ & 5.841E-02 & $1.246 \mathrm{E}-02$ \\
\hline & Mean & $1.406 \mathrm{E}+00$ & $1.123 \mathrm{E}+00$ & $1.191 \mathrm{E}+00$ & $8.675 \mathrm{E}-01$ \\
\hline & Std. & $3.961 \mathrm{E}-01$ & $5.266 \mathrm{E}-01$ & $4.356 \mathrm{E}-01$ & $6.106 \mathrm{E}-01$ \\
\hline \multirow{3}{*}{$f^{9}$} & Best & $1.038 \mathrm{E}+00$ & $9.870 \mathrm{E}-01$ & $1.055 \mathrm{E}+00$ & $2.596 \mathrm{E}-01$ \\
\hline & Mean & $1.124 \mathrm{E}+00$ & $1.090 \mathrm{E}+00$ & $1.118 \mathrm{E}+00$ & $1.074 \mathrm{E}+00$ \\
\hline & Std. & 4.877E-02 & $4.527 \mathrm{E}-02$ & 4.242E-02 & $1.619 \mathrm{E}-01$ \\
\hline \multirow{3}{*}{$f 10$} & Best & $-1.032 E+00$ & $-1.032 E+00$ & $-1.032 E+00$ & $-1.032 E+00$ \\
\hline & Mean & $-1.032 \mathrm{E}+00$ & $-1.032 \mathrm{E}+00$ & $-1.032 \mathrm{E}+00$ & $-1.032 \mathrm{E}+00$ \\
\hline & Std. & $1.698 \mathrm{E}-10$ & $1.083 \mathrm{E}-10$ & $1.832 \mathrm{E}-10$ & $3.244 \mathrm{E}-10$ \\
\hline \multirow{3}{*}{$f 11$} & Best & $6.237 \mathrm{E}+00$ & $1.280 \mathrm{E}-02$ & $3.949 \mathrm{E}+00$ & $7.787 \mathrm{E}+00$ \\
\hline & Mean & $1.240 \mathrm{E}+01$ & $1.958 \mathrm{E}+00$ & $9.605 \mathrm{E}+00$ & $9.684 \mathrm{E}+03$ \\
\hline & Std. & $4.391 \mathrm{E}+00$ & $3.266 \mathrm{E}+00$ & $4.487 \mathrm{E}+00$ & $2.235 \mathrm{E}+04$ \\
\hline
\end{tabular}

(continued) 


\begin{tabular}{llcccc}
\hline $\begin{array}{c}\text { Benchmark } \\
\text { function }\end{array}$ & & $\boldsymbol{O H S A}$ & $\boldsymbol{G H S A}$ & $\boldsymbol{T H S A}$ & VSM \\
\hline \multirow{3}{*}{$\boldsymbol{f 1 2}$} & Best & $\mathbf{4 . 8 3 2 E}+\mathbf{0 3}$ & $4.866 \mathrm{E}+03$ & $6.103 \mathrm{E}+03$ & $1.051 \mathrm{E}+04$ \\
& Mean & $1.077 \mathrm{E}+04$ & $1.013 \mathrm{E}+04$ & $1.004 \mathrm{E}+04$ & $2.100 \mathrm{E}+04$ \\
& Std. & $3.205 \mathrm{E}+03$ & $2.263 \mathrm{E}+03$ & $2.356 \mathrm{E}+03$ & $1.366 \mathrm{E}+04$ \\
\hline \multirow{3}{*13}{} & Best & $1.826 \mathrm{E}+03$ & $\mathbf{3 . 3 4 8 E}+\mathbf{0 2}$ & $1.452 \mathrm{E}+03$ & $1.304 \mathrm{E}+03$ \\
& Mean & $1.072 \mathrm{E}+04$ & $2.777 \mathrm{E}+03$ & $5.821 \mathrm{E}+03$ & $7.900 \mathrm{E}+08$ \\
& Std. & $7.156 \mathrm{E}+03$ & $4.255 \mathrm{E}+03$ & $4.034 \mathrm{E}+03$ & $4.327 \mathrm{E}+09$ \\
\hline \multirow{3}{*}{$\boldsymbol{f 4}$} & Best & $4.260 \mathrm{E}-02$ & $3.157 \mathrm{E}-02$ & $3.569 \mathrm{E}-02$ & $\mathbf{2 . 6 9 1 E - 0 2}$ \\
& Mean & $1.293 \mathrm{E}+00$ & $8.915 \mathrm{E}-01$ & $1.082 \mathrm{E}+00$ & $8.658 \mathrm{E}+00$ \\
& Std. & $1.248 \mathrm{E}+00$ & $9.407 \mathrm{E}-01$ & $1.020 \mathrm{E}+00$ & $3.804 \mathrm{E}+01$ \\
\hline
\end{tabular}

The observed mean results show that the VSM achieves the best mean score in $50 \%, 43 \%$, and $29 \%$ of total cases in 5, 20, and 50 HMS, respectively, among the compared algorithms.

The high standard deviation values obtained by the VSM in all cases of $f 11$ and $f 13$ test functions indicate the instability of the VSM search progress.

Table 4

Best, Mean and Standard Deviation of the Benchmark Function Results $(H M S=50, N D=30)$

\begin{tabular}{|c|c|c|c|c|c|}
\hline \multicolumn{2}{|c|}{$\begin{array}{l}\text { Benchmark } \\
\text { function }\end{array}$} & \multirow{2}{*}{$\frac{\boldsymbol{O H S A}}{7.501 \mathrm{E}+00}$} & \multirow{2}{*}{$\frac{G H S A}{2.136 \mathrm{E}-01}$} & \multirow{2}{*}{$\frac{\boldsymbol{T H S A}}{6.420 \mathrm{E}+00}$} & \multirow{2}{*}{$\frac{\text { VSM }}{3.592 \mathrm{E}-01}$} \\
\hline \multirow{3}{*}{$f 1$} & Best & & & & \\
\hline & Mean & $2.156 \mathrm{E}+01$ & $1.983 \mathrm{E}+00$ & $1.570 \mathrm{E}+01$ & $1.153 \mathrm{E}+01$ \\
\hline & Std. & $7.203 \mathrm{E}+00$ & $1.973 \mathrm{E}+00$ & $6.269 \mathrm{E}+00$ & $5.905 \mathrm{E}+00$ \\
\hline \multirow{3}{*}{$f^{2}$} & Best & 2.499E-01 & $4.963 \mathrm{E}-02$ & 7.990E-02 & $5.933 \mathrm{E}-02$ \\
\hline & Mean & 4.992E-01 & $7.621 \mathrm{E}-02$ & $2.426 \mathrm{E}-01$ & $2.636 \mathrm{E}-01$ \\
\hline & Std. & $1.802 \mathrm{E}-01$ & $5.257 \mathrm{E}-02$ & $1.148 \mathrm{E}-01$ & $1.679 \mathrm{E}-01$ \\
\hline \multirow{3}{*}{$f 3$} & Best & $2.000 \mathrm{E}+00$ & $0.000 \mathrm{E}+00$ & $0.000 \mathrm{E}+00$ & $0.000 E+00$ \\
\hline & Mean & $9.533 \mathrm{E}+00$ & $3.567 \mathrm{E}+00$ & $6.733 \mathrm{E}+00$ & $5.333 \mathrm{E}+00$ \\
\hline & Std. & $5.070 \mathrm{E}+00$ & $2.329 \mathrm{E}+00$ & $4.085 \mathrm{E}+00$ & $4.505 \mathrm{E}+00$ \\
\hline \multirow{3}{*}{ f4 } & Best & $2.606 \mathrm{E}+02$ & $1.868 E+01$ & $2.165 \mathrm{E}+02$ & $1.049 \mathrm{E}+02$ \\
\hline & Mean & $8.307 \mathrm{E}+02$ & $2.240 \mathrm{E}+02$ & $5.518 \mathrm{E}+02$ & $3.538 \mathrm{E}+02$ \\
\hline & Std. & $5.765 \mathrm{E}+02$ & $2.743 \mathrm{E}+02$ & $4.119 \mathrm{E}+02$ & $2.365 \mathrm{E}+02$ \\
\hline \multirow{3}{*}{$f 5$} & Best & $2.840 \mathrm{E}+03$ & $3.835 \mathrm{E}+03$ & $2.468 \mathrm{E}+03$ & $1.041 \mathrm{E}+02$ \\
\hline & Mean & $8.822 \mathrm{E}+03$ & $8.882 \mathrm{E}+03$ & $6.415 \mathrm{E}+03$ & $1.399 \mathrm{E}+03$ \\
\hline & Std. & $3.591 \mathrm{E}+03$ & $2.476 \mathrm{E}+03$ & $2.374 \mathrm{E}+03$ & $1.456 \mathrm{E}+03$ \\
\hline \multirow{3}{*}{ f6 } & Best & $-1.255 \mathrm{E}+04$ & $-1.256 \mathrm{E}+04$ & $-1.255 \mathrm{E}+04$ & $-1.257 E+04$ \\
\hline & Mean & $-1.252 \mathrm{E}+04$ & $-1.254 \mathrm{E}+04$ & $-1.253 \mathrm{E}+04$ & $-1.253 \mathrm{E}+04$ \\
\hline & Std. & $1.681 \mathrm{E}+01$ & $1.071 \mathrm{E}+01$ & $1.436 \mathrm{E}+01$ & $2.180 \mathrm{E}+01$ \\
\hline \multirow{3}{*}{$f 7$} & Best & $1.233 \mathrm{E}-01$ & $4.518 \mathrm{E}-02$ & $5.418 \mathrm{E}-02$ & 3.989E-02 \\
\hline & Mean & $1.760 \mathrm{E}+00$ & $1.028 \mathrm{E}+00$ & $1.521 \mathrm{E}+00$ & $2.090 \mathrm{E}+00$ \\
\hline & Std. & $1.186 \mathrm{E}+00$ & $8.751 \mathrm{E}-01$ & $1.492 \mathrm{E}+00$ & $5.347 \mathrm{E}+00$ \\
\hline \multirow{3}{*}{$f 8$} & Best & $1.264 \mathrm{E}+00$ & $1.066 \mathrm{E}-02$ & $8.157 \mathrm{E}-01$ & $7.178 \mathrm{E}-02$ \\
\hline & Mean & $2.071 \mathrm{E}+00$ & $1.014 \mathrm{E}+00$ & $1.736 \mathrm{E}+00$ & $1.226 \mathrm{E}+00$ \\
\hline & Std. & $3.663 \mathrm{E}-01$ & $6.125 \mathrm{E}-01$ & 4.419E-01 & $6.251 \mathrm{E}-01$ \\
\hline
\end{tabular}

(continued) 


\begin{tabular}{|c|c|c|c|c|c|}
\hline \multicolumn{2}{|c|}{$\begin{array}{l}\text { Benchmark } \\
\text { function }\end{array}$} & $O H S A$ & $G H S A$ & $T H S A$ & VSM \\
\hline \multirow{3}{*}{$f^{9}$} & Best & $1.099 \mathrm{E}+00$ & $9.392 \mathrm{E}-01$ & $1.056 \mathrm{E}+00$ & $3.935 \mathrm{E}-01$ \\
\hline & Mean & $1.214 \mathrm{E}+00$ & $1.077 \mathrm{E}+00$ & $1.127 \mathrm{E}+00$ & $1.087 \mathrm{E}+00$ \\
\hline & Std. & $6.325 \mathrm{E}-02$ & 4.684E-02 & $4.369 \mathrm{E}-02$ & $1.801 \mathrm{E}-01$ \\
\hline \multirow{3}{*}{$f 10$} & Best & $-1.032 E+00$ & $-1.032 E+00$ & $-1.032 E+00$ & $-1.032 E+00$ \\
\hline & Mean & $-1.032 \mathrm{E}+00$ & $-1.032 \mathrm{E}+00$ & $-1.032 \mathrm{E}+00$ & $-1.032 \mathrm{E}+00$ \\
\hline & Std. & $1.338 \mathrm{E}-10$ & $2.879 \mathrm{E}-10$ & $3.567 \mathrm{E}-10$ & $2.766 \mathrm{E}-10$ \\
\hline \multirow{3}{*}{$f 11$} & Best & $9.933 \mathrm{E}+00$ & 9.689E-02 & $7.476 \mathrm{E}+00$ & $1.522 \mathrm{E}+01$ \\
\hline & Mean & $2.094 \mathrm{E}+01$ & $1.640 \mathrm{E}+00$ & $1.501 \mathrm{E}+01$ & $6.519 \mathrm{E}+03$ \\
\hline & Std. & $5.366 \mathrm{E}+00$ & $1.709 \mathrm{E}+00$ & $5.314 \mathrm{E}+00$ & $1.782 \mathrm{E}+04$ \\
\hline \multirow{3}{*}{$f 12$} & Best & $5.124 \mathrm{E}+03$ & $5.094 \mathrm{E}+03$ & $4.789 \mathrm{E}+03$ & $4.068 \mathrm{E}+03$ \\
\hline & Mean & $1.039 \mathrm{E}+04$ & $1.028 \mathrm{E}+04$ & $9.305 \mathrm{E}+03$ & $1.805 \mathrm{E}+04$ \\
\hline & Std. & $2.771 \mathrm{E}+03$ & $3.734 \mathrm{E}+03$ & $2.337 \mathrm{E}+03$ & $9.635 \mathrm{E}+03$ \\
\hline \multirow{3}{*}{$f 13$} & Best & $3.947 \mathrm{E}+03$ & $4.852 \mathrm{E}+02$ & $1.776 E+03$ & $4.982 \mathrm{E}+03$ \\
\hline & Mean & $1.745 \mathrm{E}+04$ & $2.278 \mathrm{E}+03$ & $1.293 \mathrm{E}+04$ & $7.704 \mathrm{E}+08$ \\
\hline & Std. & $8.877 \mathrm{E}+03$ & $3.040 \mathrm{E}+03$ & $8.160 \mathrm{E}+03$ & $3.480 \mathrm{E}+09$ \\
\hline \multirow{3}{*}{$f 14$} & Best & $1.836 \mathrm{E}-01$ & 2.877 E-02 & $6.993 \mathrm{E}-02$ & $2.447 \mathrm{E}-01$ \\
\hline & Mean & $2.432 \mathrm{E}+00$ & $9.694 \mathrm{E}-01$ & $1.612 \mathrm{E}+00$ & $1.651 \mathrm{E}+01$ \\
\hline & Std. & $1.481 \mathrm{E}+00$ & $1.048 \mathrm{E}+00$ & $1.102 \mathrm{E}+00$ & $4.258 \mathrm{E}+01$ \\
\hline
\end{tabular}

This result is attributed to the weak performance of the VSM procedure in detecting promising divisions in these two functions, leading to a relatively slow convergence rate. Note that the evaluation number used in the detection procedure is considered and deducted from the amount of remaining evaluation times.

Tables 5, 6, and 7 show the statistical results obtained on the basis of the solution fitness value for 100 problem dimensions.

Table 5

Best, Mean and Standard Deviation of the Benchmark Function Results $(H M S=5, N D=100)$

\begin{tabular}{|c|c|c|c|c|c|}
\hline $\begin{array}{l}\text { Benchmark } \\
\text { function }\end{array}$ & & $O H S A$ & $G H S A$ & THSA & VSM \\
\hline & Best & $4.852 \mathrm{E}+03$ & $3.698 \mathrm{E}+03$ & $4.603 E+03$ & $8.038 E+01$ \\
\hline \multirow[t]{2}{*}{$f 1$} & Mean & $6.443 \mathrm{E}+03$ & $5.983 \mathrm{E}+03$ & $5.800 \mathrm{E}+03$ & $1.413 \mathrm{E}+03$ \\
\hline & Std. & $8.404 \mathrm{E}+02$ & $9.638 \mathrm{E}+02$ & $8.185 \mathrm{E}+02$ & $1.654 \mathrm{E}+03$ \\
\hline \multirow{3}{*}{$f^{2}$} & Best & $3.700 \mathrm{E}+01$ & $4.144 \mathrm{E}+01$ & $4.064 \mathrm{E}+01$ & $9.800 \mathrm{E}+00$ \\
\hline & Mean & $4.710 \mathrm{E}+01$ & $4.672 \mathrm{E}+01$ & $4.876 \mathrm{E}+01$ & $2.325 \mathrm{E}+01$ \\
\hline & Std. & $3.779 \mathrm{E}+00$ & $3.174 \mathrm{E}+00$ & $3.815 \mathrm{E}+00$ & $1.182 \mathrm{E}+01$ \\
\hline \multirow{3}{*}{$f 3$} & Best & $4.819 \mathrm{E}+03$ & $3.976 \mathrm{E}+03$ & $3.989 \mathrm{E}+03$ & $1.180 \mathrm{E}+02$ \\
\hline & Mean & $5.825 \mathrm{E}+03$ & $5.429 \mathrm{E}+03$ & $5.400 \mathrm{E}+03$ & $1.301 \mathrm{E}+03$ \\
\hline & Std. & $7.527 \mathrm{E}+02$ & $7.599 \mathrm{E}+02$ & $8.274 \mathrm{E}+02$ & $1.451 \mathrm{E}+03$ \\
\hline \multirow{3}{*}{ f4 } & Best & $9.182 \mathrm{E}+05$ & $7.031 \mathrm{E}+05$ & $3.953 \mathrm{E}+05$ & $1.921 \mathrm{E}+03$ \\
\hline & Mean & $1.513 \mathrm{E}+06$ & $1.383 \mathrm{E}+06$ & $1.084 \mathrm{E}+06$ & $2.360 \mathrm{E}+05$ \\
\hline & Std. & $3.349 \mathrm{E}+05$ & $3.863 \mathrm{E}+05$ & $3.942 \mathrm{E}+05$ & $5.333 \mathrm{E}+05$ \\
\hline
\end{tabular}




\begin{tabular}{|c|c|c|c|c|c|}
\hline $\begin{array}{l}\text { Benchmark } \\
\text { function }\end{array}$ & & OHSA & GHSA & THSA & VSM \\
\hline \multirow{3}{*}{$f 5$} & Best & $1.154 \mathrm{E}+05$ & $1.233 \mathrm{E}+05$ & $1.021 \mathrm{E}+05$ & $2.499 \mathrm{E}+03$ \\
\hline & Mean & $1.596 \mathrm{E}+05$ & $1.522 \mathrm{E}+05$ & $1.402 \mathrm{E}+05$ & $5.197 \mathrm{E}+04$ \\
\hline & Std. & $2.722 \mathrm{E}+04$ & $1.664 \mathrm{E}+04$ & $1.779 \mathrm{E}+04$ & $8.843 \mathrm{E}+04$ \\
\hline \multirow{3}{*}{ f6 } & Best & $-3.791 \mathrm{E}+04$ & $-3.774 \mathrm{E}+04$ & $-3.693 \mathrm{E}+04$ & $-4.164 E+04$ \\
\hline & Mean & $-3.689 \mathrm{E}+04$ & $-3.677 \mathrm{E}+04$ & $-3.600 \mathrm{E}+04$ & $-3.963 E+04$ \\
\hline & Std. & $5.016 \mathrm{E}+02$ & $6.163 \mathrm{E}+02$ & $6.005 \mathrm{E}+02$ & $1.484 \mathrm{E}+03$ \\
\hline \multirow{3}{*}{$f 7$} & Best & $1.800 \mathrm{E}+02$ & $1.765 \mathrm{E}+02$ & $1.751 \mathrm{E}+02$ & $5.788 E+01$ \\
\hline & Mean & $2.047 \mathrm{E}+02$ & $1.977 \mathrm{E}+02$ & $2.083 E+02$ & $1.260 \mathrm{E}+02$ \\
\hline & Std. & $1.425 \mathrm{E}+01$ & $1.298 \mathrm{E}+01$ & $1.863 \mathrm{E}+01$ & $4.468 \mathrm{E}+01$ \\
\hline \multirow{3}{*}{ f8 } & Best & $8.298 \mathrm{E}+00$ & $8.436 \mathrm{E}+00$ & $8.322 \mathrm{E}+00$ & $3.413 \mathrm{E}+00$ \\
\hline & Mean & $9.534 \mathrm{E}+00$ & $9.484 \mathrm{E}+00$ & $9.327 \mathrm{E}+00$ & $5.679 \mathrm{E}+00$ \\
\hline & Std. & 4.712E-01 & $4.436 \mathrm{E}-01$ & $4.931 \mathrm{E}-01$ & $2.166 \mathrm{E}+00$ \\
\hline \multirow{3}{*}{$f^{9}$} & Best & $4.118 \mathrm{E}+01$ & $4.502 \mathrm{E}+01$ & $3.913 \mathrm{E}+01$ & $1.734 E+00$ \\
\hline & Mean & $5.790 \mathrm{E}+01$ & $5.754 \mathrm{E}+01$ & $5.362 \mathrm{E}+01$ & $1.607 \mathrm{E}+01$ \\
\hline & Std. & $7.542 \mathrm{E}+00$ & $6.802 \mathrm{E}+00$ & $8.945 \mathrm{E}+00$ & $1.689 \mathrm{E}+01$ \\
\hline \multirow{3}{*}{$f 10$} & Best & $-1.032 E+00$ & $-1.032 E+00$ & $-1.032 E+00$ & $-1.032 E+00$ \\
\hline & Mean & $-1.032 \mathrm{E}+00$ & $-1.032 \mathrm{E}+00$ & $-1.032 \mathrm{E}+00$ & $-1.032 \mathrm{E}+00$ \\
\hline & Std. & $3.469 \mathrm{E}-10$ & $1.838 \mathrm{E}-10$ & $2.965 \mathrm{E}-10$ & $2.247 \mathrm{E}-10$ \\
\hline \multirow{3}{*}{$f 11$} & Best & $4.658 E+03$ & $4.930 \mathrm{E}+03$ & $5.012 \mathrm{E}+03$ & $5.808 \mathrm{E}+03$ \\
\hline & Mean & $6.952 E+03$ & $6.297 \mathrm{E}+03$ & $6.405 E+03$ & $3.522 \mathrm{E}+04$ \\
\hline & Std. & $1.209 \mathrm{E}+03$ & $8.616 \mathrm{E}+02$ & $9.371 \mathrm{E}+02$ & $5.916 \mathrm{E}+04$ \\
\hline \multirow{3}{*}{$f 12$} & Best & $1.586 \mathrm{E}+05$ & $1.680 \mathrm{E}+05$ & $1.358 \mathrm{E}+05$ & $1.282 \mathrm{E}+05$ \\
\hline & Mean & $2.160 \mathrm{E}+05$ & $2.116 \mathrm{E}+05$ & $1.929 \mathrm{E}+05$ & $1.975 \mathrm{E}+05$ \\
\hline & Std. & $2.938 \mathrm{E}+04$ & $2.929 \mathrm{E}+04$ & $2.794 \mathrm{E}+04$ & $8.819 \mathrm{E}+04$ \\
\hline \multirow{3}{*}{$f 13$} & Best & $1.001 \mathrm{E}+08$ & $1.135 \mathrm{E}+08$ & $6.670 \mathrm{E}+07$ & $1.258 \mathrm{E}+08$ \\
\hline & Mean & $2.071 \mathrm{E}+08$ & $1.764 \mathrm{E}+08$ & $1.329 \mathrm{E}+08$ & $8.750 \mathrm{E}+09$ \\
\hline & Std. & $6.028 \mathrm{E}+07$ & $4.782 \mathrm{E}+07$ & $3.409 \mathrm{E}+07$ & $2.095 \mathrm{E}+10$ \\
\hline \multirow{3}{*}{ f14 } & Best & $1.985 \mathrm{E}+02$ & $1.911 \mathrm{E}+02$ & $1.860 \mathrm{E}+02$ & $2.008 \mathrm{E}+02$ \\
\hline & Mean & $2.216 \mathrm{E}+02$ & $2.218 \mathrm{E}+02$ & $2.244 \mathrm{E}+02$ & $2.772 \mathrm{E}+02$ \\
\hline & Std. & $1.272 \mathrm{E}+01$ & $1.568 \mathrm{E}+01$ & $1.709 \mathrm{E}+01$ & $1.185 \mathrm{E}+02$ \\
\hline
\end{tabular}

The observed results show that the VSM has excellent performance in $100 \%$ of the cases of non-shifted functions and acceptable performance in $25 \%$ of the cases of shifted functions.

Moreover, the observed mean results show comparable performance between the compared algorithms where the proposed technique achieves the best mean score in $71 \%, 71 \%$, and $64 \%$ of total experimental cases for 5,20 and $50 \mathrm{HMS}$, respectively. 
Table 6

Best, Mean and Standard Deviation of the Benchmark Sunction Results $(H M S=20, N D=100)$

\begin{tabular}{|c|c|c|c|c|c|}
\hline $\begin{array}{l}\text { Benchmark } \\
\text { function }\end{array}$ & & $O H S A$ & $G H S A$ & THSA & VSM \\
\hline & Best & $5.002 \mathrm{E}+03$ & $5.192 \mathrm{E}+03$ & $4.447 \mathrm{E}+03$ & $1.147 \mathrm{E}+02$ \\
\hline \multirow[t]{2}{*}{$f 1$} & Mean & $7.327 \mathrm{E}+03$ & $7.230 \mathrm{E}+03$ & $5.888 \mathrm{E}+03$ & $2.634 \mathrm{E}+03$ \\
\hline & Std. & $8.831 \mathrm{E}+02$ & $1.031 \mathrm{E}+03$ & $8.772 \mathrm{E}+02$ & $2.871 \mathrm{E}+03$ \\
\hline \multirow{3}{*}{$f^{2}$} & Best & $3.643 \mathrm{E}+01$ & $3.734 \mathrm{E}+01$ & $4.424 \mathrm{E}+01$ & $4.465 E+00$ \\
\hline & Mean & $4.272 \mathrm{E}+01$ & $4.331 \mathrm{E}+01$ & $5.004 \mathrm{E}+01$ & $2.347 \mathrm{E}+01$ \\
\hline & Std. & $3.230 \mathrm{E}+00$ & $3.004 \mathrm{E}+00$ & $3.564 \mathrm{E}+00$ & $1.309 \mathrm{E}+01$ \\
\hline \multirow{3}{*}{$f 3$} & Best & $5.343 \mathrm{E}+03$ & $5.546 \mathrm{E}+03$ & $4.170 \mathrm{E}+03$ & $1.260 \mathrm{E}+02$ \\
\hline & Mean & $6.885 \mathrm{E}+03$ & $6.815 \mathrm{E}+03$ & $5.405 \mathrm{E}+03$ & $1.373 \mathrm{E}+03$ \\
\hline & Std. & $8.548 \mathrm{E}+02$ & $8.218 \mathrm{E}+02$ & $7.593 \mathrm{E}+02$ & $1.676 \mathrm{E}+03$ \\
\hline \multirow{3}{*}{$f 4$} & Best & $2.000 \mathrm{E}+06$ & $1.204 \mathrm{E}+06$ & $6.347 \mathrm{E}+05$ & $2.781 \mathrm{E}+03$ \\
\hline & Mean & $2.850 \mathrm{E}+06$ & $2.290 \mathrm{E}+06$ & $1.077 \mathrm{E}+06$ & $6.131 \mathrm{E}+05$ \\
\hline & Std. & $5.631 \mathrm{E}+05$ & $4.844 \mathrm{E}+05$ & $3.166 \mathrm{E}+05$ & $1.105 \mathrm{E}+06$ \\
\hline \multirow{3}{*}{ f5 } & Best & $2.235 \mathrm{E}+05$ & $1.653 \mathrm{E}+05$ & $1.170 \mathrm{E}+05$ & $5.811 E+03$ \\
\hline & Mean & $3.045 \mathrm{E}+05$ & $2.269 \mathrm{E}+05$ & $1.478 \mathrm{E}+05$ & $5.804 \mathrm{E}+05$ \\
\hline & Std. & $5.787 \mathrm{E}+04$ & $4.188 \mathrm{E}+04$ & $1.736 \mathrm{E}+04$ & $1.554 \mathrm{E}+06$ \\
\hline \multirow{3}{*}{ f6 } & Best & $-3.843 \mathrm{E}+04$ & $-3.880 \mathrm{E}+04$ & $-3.706 \mathrm{E}+04$ & $-4.163 E+04$ \\
\hline & Mean & $-3.765 \mathrm{E}+04$ & $-3.742 \mathrm{E}+04$ & $-3.605 E+04$ & $-3.985 E+04$ \\
\hline & Std. & $5.605 \mathrm{E}+02$ & $5.260 \mathrm{E}+02$ & $6.157 \mathrm{E}+02$ & $1.190 \mathrm{E}+03$ \\
\hline \multirow{3}{*}{$f 7$} & Best & $1.605 \mathrm{E}+02$ & $1.736 \mathrm{E}+02$ & $1.863 \mathrm{E}+02$ & $8.332 \mathrm{E}+01$ \\
\hline & Mean & $1.864 \mathrm{E}+02$ & $1.952 \mathrm{E}+02$ & $2.151 \mathrm{E}+02$ & $1.394 \mathrm{E}+02$ \\
\hline & Std. & $1.581 \mathrm{E}+01$ & $1.362 \mathrm{E}+01$ & $1.557 \mathrm{E}+01$ & $4.122 \mathrm{E}+01$ \\
\hline \multirow{3}{*}{ f8 } & Best & $8.785 E+00$ & $9.163 \mathrm{E}+00$ & $8.382 \mathrm{E}+00$ & $2.137 \mathrm{E}+00$ \\
\hline & Mean & $1.006 \mathrm{E}+01$ & $9.935 \mathrm{E}+00$ & $9.340 \mathrm{E}+00$ & $5.401 \mathrm{E}+00$ \\
\hline & Std. & $3.721 \mathrm{E}-01$ & $4.087 \mathrm{E}-01$ & 4.417E-01 & $2.160 \mathrm{E}+00$ \\
\hline \multirow{3}{*}{$f^{9}$} & Best & $5.083 \mathrm{E}+01$ & $5.161 \mathrm{E}+01$ & $4.332 \mathrm{E}+01$ & $2.452 \mathrm{E}+00$ \\
\hline & Mean & $6.959 \mathrm{E}+01$ & $6.365 \mathrm{E}+01$ & $5.503 \mathrm{E}+01$ & $2.397 \mathrm{E}+01$ \\
\hline & Std. & $1.035 \mathrm{E}+01$ & $9.249 \mathrm{E}+00$ & $9.756 \mathrm{E}+00$ & $2.501 \mathrm{E}+01$ \\
\hline \multirow{3}{*}{$f 10$} & Best & $-1.032 E+00$ & $-1.032 E+00$ & $-1.032 E+00$ & $-1.032 E+00$ \\
\hline & Mean & $-1.032 \mathrm{E}+00$ & $-1.032 \mathrm{E}+00$ & $-1.032 \mathrm{E}+00$ & $-1.032 \mathrm{E}+00$ \\
\hline & Std. & $2.528 \mathrm{E}-10$ & $4.286 \mathrm{E}-10$ & $1.903 \mathrm{E}-10$ & $1.279 \mathrm{E}-10$ \\
\hline \multirow{3}{*}{$f 11$} & Best & $6.874 \mathrm{E}+03$ & $5.880 \mathrm{E}+03$ & $3.825 E+03$ & $6.941 \mathrm{E}+03$ \\
\hline & Mean & $8.323 \mathrm{E}+03$ & $7.805 \mathrm{E}+03$ & $5.898 \mathrm{E}+03$ & $4.558 \mathrm{E}+04$ \\
\hline & Std. & $9.388 \mathrm{E}+02$ & $1.001 \mathrm{E}+03$ & $8.438 \mathrm{E}+02$ & $6.820 \mathrm{E}+04$ \\
\hline \multirow{3}{*}{$f 12$} & Best & $2.497 \mathrm{E}+05$ & $1.962 \mathrm{E}+05$ & $1.311 \mathrm{E}+05$ & $1.419 \mathrm{E}+05$ \\
\hline & Mean & $3.427 \mathrm{E}+05$ & $2.700 \mathrm{E}+05$ & $1.925 \mathrm{E}+05$ & $2.189 \mathrm{E}+05$ \\
\hline & Std. & $4.207 \mathrm{E}+04$ & $3.729 \mathrm{E}+04$ & $3.002 \mathrm{E}+04$ & $8.858 \mathrm{E}+04$ \\
\hline \multirow{3}{*}{$f 13$} & Best & $2.851 \mathrm{E}+08$ & $1.840 \mathrm{E}+08$ & $6.882 E+07$ & $3.943 \mathrm{E}+08$ \\
\hline & Mean & $4.204 \mathrm{E}+08$ & $3.064 \mathrm{E}+08$ & $1.303 \mathrm{E}+08$ & $4.754 \mathrm{E}+09$ \\
\hline & Std. & $8.033 \mathrm{E}+07$ & $7.245 \mathrm{E}+07$ & $3.490 \mathrm{E}+07$ & $1.607 \mathrm{E}+10$ \\
\hline \multirow{3}{*}{ f14 } & Best & $1.791 \mathrm{E}+02$ & $1.743 E+02$ & $1.846 \mathrm{E}+02$ & $1.891 \mathrm{E}+02$ \\
\hline & Mean & $2.071 \mathrm{E}+02$ & $2.161 \mathrm{E}+02$ & $2.227 \mathrm{E}+02$ & $2.935 \mathrm{E}+02$ \\
\hline & Std. & $1.489 \mathrm{E}+01$ & $1.914 \mathrm{E}+01$ & $2.031 \mathrm{E}+01$ & $1.781 \mathrm{E}+02$ \\
\hline
\end{tabular}


Table 7

Best, Mean and Standard dDviation of the Benchmark Function Results $(H M S=50, N D=100)$

\begin{tabular}{|c|c|c|c|c|c|}
\hline $\begin{array}{l}\text { Benchmark } \\
\text { function }\end{array}$ & & OHSA & GHSA & THSA & VSM \\
\hline & Best & $4.852 \mathrm{E}+03$ & $3.698 \mathrm{E}+03$ & $4.603 \mathrm{E}+03$ & $8.038 E+01$ \\
\hline \multirow[t]{2}{*}{$f 1$} & Mean & $6.443 \mathrm{E}+03$ & $5.983 \mathrm{E}+03$ & $5.800 \mathrm{E}+03$ & $1.413 \mathrm{E}+03$ \\
\hline & Std. & $8.404 \mathrm{E}+02$ & $9.638 \mathrm{E}+02$ & $8.185 \mathrm{E}+02$ & $1.654 \mathrm{E}+03$ \\
\hline \multirow{3}{*}{$f^{2}$} & Best & $3.700 \mathrm{E}+01$ & $4.144 \mathrm{E}+01$ & $4.064 \mathrm{E}+01$ & $9.800 \mathrm{E}+00$ \\
\hline & Mean & $4.710 \mathrm{E}+01$ & $4.672 \mathrm{E}+01$ & $4.876 \mathrm{E}+01$ & $2.325 \mathrm{E}+01$ \\
\hline & Std. & $3.779 \mathrm{E}+00$ & $3.174 \mathrm{E}+00$ & $3.815 \mathrm{E}+00$ & $1.182 \mathrm{E}+01$ \\
\hline \multirow{3}{*}{$f 3$} & Best & $4.819 \mathrm{E}+03$ & $3.976 \mathrm{E}+03$ & $3.989 \mathrm{E}+03$ & $1.180 \mathrm{E}+02$ \\
\hline & Mean & $5.825 \mathrm{E}+03$ & $5.429 \mathrm{E}+03$ & $5.400 \mathrm{E}+03$ & $1.301 \mathrm{E}+03$ \\
\hline & Std. & $7.527 \mathrm{E}+02$ & $7.599 \mathrm{E}+02$ & $8.274 \mathrm{E}+02$ & $1.451 \mathrm{E}+03$ \\
\hline \multirow{3}{*}{ f4 } & Best & $9.182 \mathrm{E}+05$ & $7.031 \mathrm{E}+05$ & $3.953 \mathrm{E}+05$ & $1.921 \mathrm{E}+03$ \\
\hline & Mean & $1.513 \mathrm{E}+06$ & $1.383 \mathrm{E}+06$ & $1.084 \mathrm{E}+06$ & $2.360 \mathrm{E}+05$ \\
\hline & Std. & $3.349 \mathrm{E}+05$ & $3.863 \mathrm{E}+05$ & $3.942 \mathrm{E}+05$ & $5.333 \mathrm{E}+05$ \\
\hline \multirow{3}{*}{$f 5$} & Best & $1.154 \mathrm{E}+05$ & $1.233 \mathrm{E}+05$ & $1.021 \mathrm{E}+05$ & $2.499 \mathrm{E}+03$ \\
\hline & Mean & $1.596 \mathrm{E}+05$ & $1.522 \mathrm{E}+05$ & $1.402 \mathrm{E}+05$ & $5.197 \mathrm{E}+04$ \\
\hline & Std. & $2.722 \mathrm{E}+04$ & $1.664 \mathrm{E}+04$ & $1.779 \mathrm{E}+04$ & $8.843 \mathrm{E}+04$ \\
\hline \multirow{3}{*}{ f6 } & Best & $-3.791 \mathrm{E}+04$ & $-3.774 \mathrm{E}+04$ & $-3.693 \mathrm{E}+04$ & $-4.164 E+04$ \\
\hline & Mean & $-3.689 \mathrm{E}+04$ & $-3.677 \mathrm{E}+04$ & $-3.600 \mathrm{E}+04$ & $-3.963 \mathrm{E}+04$ \\
\hline & Std. & $5.016 \mathrm{E}+02$ & $6.163 \mathrm{E}+02$ & $6.005 \mathrm{E}+02$ & $1.484 \mathrm{E}+03$ \\
\hline \multirow{3}{*}{$f^{7}$} & Best & $1.800 \mathrm{E}+02$ & $1.765 \mathrm{E}+02$ & $1.751 \mathrm{E}+02$ & $5.788 E+01$ \\
\hline & Mean & $2.047 \mathrm{E}+02$ & $1.977 \mathrm{E}+02$ & $2.083 \mathrm{E}+02$ & $1.260 \mathrm{E}+02$ \\
\hline & Std. & $1.425 \mathrm{E}+01$ & $1.298 \mathrm{E}+01$ & $1.863 \mathrm{E}+01$ & $4.468 \mathrm{E}+01$ \\
\hline \multirow{3}{*}{$f 8$} & Best & $8.298 \mathrm{E}+00$ & $8.436 \mathrm{E}+00$ & $8.322 \mathrm{E}+00$ & $3.413 E+00$ \\
\hline & Mean & $9.534 \mathrm{E}+00$ & $9.484 \mathrm{E}+00$ & $9.327 \mathrm{E}+00$ & $5.679 \mathrm{E}+00$ \\
\hline & Std. & $4.712 \mathrm{E}-01$ & 4.436E-01 & $4.931 \mathrm{E}-01$ & $2.166 \mathrm{E}+00$ \\
\hline \multirow{3}{*}{$f^{9}$} & Best & $4.118 \mathrm{E}+01$ & $4.502 \mathrm{E}+01$ & $3.913 \mathrm{E}+01$ & $1.734 E+00$ \\
\hline & Mean & $5.790 \mathrm{E}+01$ & $5.754 \mathrm{E}+01$ & $5.362 \mathrm{E}+01$ & $1.607 \mathrm{E}+01$ \\
\hline & Std. & $7.542 \mathrm{E}+00$ & $6.802 \mathrm{E}+00$ & $8.945 \mathrm{E}+00$ & $1.689 \mathrm{E}+01$ \\
\hline \multirow{3}{*}{ f10 } & Best & $-1.032 E+00$ & $-1.032 E+00$ & $-1.032 E+00$ & $-1.032 E+00$ \\
\hline & Mean & $-1.032 \mathrm{E}+00$ & $-1.032 \mathrm{E}+00$ & $-1.032 \mathrm{E}+00$ & $-1.032 \mathrm{E}+00$ \\
\hline & Std. & $3.469 \mathrm{E}-10$ & $1.838 \mathrm{E}-10$ & $2.965 \mathrm{E}-10$ & $2.247 \mathrm{E}-10$ \\
\hline \multirow{3}{*}{$f 11$} & Best & $4.658 E+03$ & $4.930 \mathrm{E}+03$ & $5.012 \mathrm{E}+03$ & $5.808 \mathrm{E}+03$ \\
\hline & Mean & $6.952 \mathrm{E}+03$ & $6.297 \mathrm{E}+03$ & $6.405 \mathrm{E}+03$ & $3.522 \mathrm{E}+04$ \\
\hline & Std. & $1.209 \mathrm{E}+03$ & $8.616 \mathrm{E}+02$ & $9.371 \mathrm{E}+02$ & $5.916 \mathrm{E}+04$ \\
\hline \multirow{3}{*}{$f 12$} & Best & $1.586 \mathrm{E}+05$ & $1.680 \mathrm{E}+05$ & $1.358 \mathrm{E}+05$ & $1.282 \mathrm{E}+05$ \\
\hline & Mean & $2.160 \mathrm{E}+05$ & $2.116 \mathrm{E}+05$ & $1.929 \mathrm{E}+05$ & $1.975 \mathrm{E}+05$ \\
\hline & Std. & $2.938 \mathrm{E}+04$ & $2.929 \mathrm{E}+04$ & $2.794 \mathrm{E}+04$ & $8.819 \mathrm{E}+04$ \\
\hline \multirow{3}{*}{$f 13$} & Best & $1.001 \mathrm{E}+08$ & $1.135 \mathrm{E}+08$ & $6.670 E+07$ & $1.258 \mathrm{E}+08$ \\
\hline & Mean & $2.071 \mathrm{E}+08$ & $1.764 \mathrm{E}+08$ & $1.329 \mathrm{E}+08$ & $8.750 \mathrm{E}+09$ \\
\hline & Std. & $6.028 \mathrm{E}+07$ & $4.782 \mathrm{E}+07$ & $3.409 \mathrm{E}+07$ & $2.095 \mathrm{E}+10$ \\
\hline \multirow{3}{*}{ f14 } & Best & $1.985 \mathrm{E}+02$ & $1.911 \mathrm{E}+02$ & $1.860 \mathrm{E}+02$ & $2.008 \mathrm{E}+02$ \\
\hline & Mean & $2.216 \mathrm{E}+02$ & $2.218 \mathrm{E}+02$ & $2.244 \mathrm{E}+02$ & $2.772 \mathrm{E}+02$ \\
\hline & Std. & $1.272 \mathrm{E}+01$ & $1.568 \mathrm{E}+01$ & $1.709 \mathrm{E}+01$ & $1.185 \mathrm{E}+02$ \\
\hline
\end{tabular}




\section{CONCLUSION AND FUTURE WORK}

The size of search space greatly affects the search strategies of optimization algorithms. In this study, a novel search algorithm named vibrant search mechanism (VSM) is proposed, which divides the search space into an array of subdivisions and orients the search procedure toward the most promising division. This algorithm facilitates searching and minimizes algorithm complexity by generating better solutions.

The effectiveness of the VSM can be evaluated by applying it within a well-known evolutionary algorithm, such as the HSA. A series of numerical simulations were performed and applied on different types of benchmark optimization functions to validate the performance of the VSM. The performance of VSM was evaluated on the basis of the generated results. The evaluation results were compared with those of basic, tournament, and global HSAs.

The results indicated that the VSM is more efficient than other algorithms in non-shifted functions and less effective in shifted functions. The increased efficiency of the VSM was attributed to its capability to guide search progress toward the selection of promising divisions during the improvisation process.

This paper offers an initial investigation of the exploration part of an evolutionary algorithm. Further work will focus on boosting the selection process of created divisions and analysis of different parameter settings of the proposed algorithm.

\section{ACKNOWLEDGEMENT}

This research received no specific grant from any funding agency in the public, commercial, or not-for profit sectors.

\section{REFERENCES}

Alia, O. M. D., \& Mandava, R. (2011). The variants of the harmony search algorithm: an overview. Artificial Intelligence Review, 36(1), 49-68.

Al-Betar, M. A., Doush, I. A., Khader, A. T., \& Awadallah, M. A. (2012). Novel selection schemes for harmony search. Applied Mathematics and Computation, 218(10), 6095-6117.

Al-Betar, M. A., Awadallah, M. A., Khader, A. T., \&Bolaji, A. L. A. (2016). Tournament-based harmony search algorithm for non-convex economic load dispatch problem. Applied Soft Computing, 47, 449-459. 
Assad, A., \& Deep, K. (2016). Applications of harmony search algorithm in data mining: A survey. In Proceedings of Fifth International Conference on Soft Computing for Problem Solving (pp. 863-874). Springer Singapore.'

Carbas, S., \& Aydogdu, I. (2017). Utilization of harmony search algorithm in optimal structural design of cold-formed steel structures. In International Conference on Harmony Search Algorithm (pp. 240251). Springer, Singapore.'

Chen, J., Pan, Q. K., \& Li, J. Q. (2012). Harmony search algorithm with dynamic control parameters. Applied Mathematics and Computation, 219(2), 592-604.

Cobos, C., León, E., \& Mendoza, M. (2010). A harmony search algorithm for clustering with feature selection. RevistaFacultad de Ingeniería Universidad de Antioquia, 55, 153-164.

Contreras, J., Amaya, I., \& Correa, R. (2014). An improved variant of the conventional harmony search algorithm. Applied Mathematics and Computation, 227, 821-830.

Doush, I. A., Al-Betar, M. A., Khader, A. T., Awadallah, M. A., \& Mohammed, A. B. (2013). Analysis of takeover time and convergence rate for harmony search with novel selection methods. International Journal of Mathematical Modelling and Numerical Optimisation, 4(4), 305-322.)

Geem, Z. W. (2009). Harmony search optimisation to the pump-included water distribution network design. Civil Engineering and Environmental Systems, 26(3), 211-221.

Geem, Z. W. (2016). Artificial satellite heat pipe design using harmony search. In Harmony Search Algorithm (pp. 423-433). Springer Berlin Heidelberg.t

Geem, Z. W., Kim, J. H., \& Loganathan, G. V. (2001). A new heuristic optimization algorithm: harmony search. Simulation, 76(2), 60-68.

Hasan, B. H. F., Doush, I. A., Al Maghayreh, E., Alkhateeb, F., \& Hamdan, M. (2014). Hybridizing harmony search algorithm with different mutation operators for continuous problems. Applied Mathematics and Computation, 232, 1166-1182.

Holland,J.H. (1975). Adaptation in natural and artificial systems. The University of Michigan Press.

Ibrahim, A. O., Shamsuddin, S. M., \& Qasem, S. N. (2015). Hybrid Nsga-II optimization for improving the three-term Bp network for multiclass classification problems. Journal of Information and Communication Technology, 14, 21-38.

Kar, P., \& Swain, S. C. (2016). A harmony search-firefly algorithm based controller for damping power oscillations. In Computational Intelligence \&Communication Technology, India, 2, 351-355. doi: 10.1109/CICT.2016.75. 
Kazmi, S. A. A., Shahzad, M. K., \& Shin, D. R. (2017). Multi-objective planning techniques in distribution networks: A composite review. Energies, 10(2), 208, doi:10.3390/en10020208.

Kennedy, R. J. and Eberhart (1995). Particle swarm optimization. In Proceedings of IEEE International Conference on Neural Networks. Perth, 4, 1942-1948.

Keshtegar, B., Hao, P., Wang, Y., \& Li, Y. (2017). Optimum design of aircraft panels based on adaptive dynamic harmony search. Thin-Walled Structures, 118, 37-45.

Khalili, M., Kharrat, R., Salahshoor, K., \&Sefat, M. H. (2014). Global dynamic harmony search algorithm: GDHS. Applied Mathematics and Computation, 228, 195-219.

Kong, Z., Gao, L., Wang, L., Ge, Y., \& Li, S. (2009). On an adaptive harmony search algorithm. International Journal of Innovative Computing, Information and Control, 5(9), 2551-2560.

Kumar, V., Chhabra, J. K., \& Kumar, D. (2014). Parameter adaptive harmony search algorithm for unimodal and multimodal optimization problems. Journal of Computational Science, 5(2), 144-155.

Lenin, K., Ravindranath R., B., \& Surya, K., M. (2013). Harmony search (HS) algorithm for solving optimal reactive power dispatch problem. International Journal of Electronics and Electrical Engineering, 1(4), 269-274.

Mahdavi, M., Fesanghary, M., \& Damangir, E. (2007). An improved harmony search algorithm for solving optimization problems. Applied Mathematics and Computation, 188(2), 1567-1579.

Omran, M. G., \&Mahdavi, M. (2008). Global-best harmony search. Applied Mathematics and Computation, 198(2), 643-656.

Ouyang, H. B., Gao, L. Q., Li, S., Kong, X. Y., Wang, Q., \& Zou, D. X. (2017). Improved harmony search algorithm: LHS. Applied Soft Computing, 53, 133-167.

Q.-K. Pan, P. Suganthan, M. F. Tasgetiren, J., L. (2010). A self-adaptive global best harmony search algorithm for continuous optimization problems. Applied Mathematics and Computation 216 (3) 830-848.

Sabarinath, P., Thansekhar, M. R., \& Saravanan, R. (2015). Multiobjective optimization method based on adaptive parameter harmony search algorithm. Journal of Applied Mathematics, Article ID 165601. doi:10.1155/2015/165601.

Satapathy, P., Dhar, S., \& Dash, P. K. (2017). Stability improvement of PV-BESS diesel generator-based microgrid with a new modified harmony search-based hybrid firefly algorithm. IET Renewable Power Generation, 11(5), 566-577. 
Shambour, M. D. K. Y., Khader, A. T., Kheiri, A., \& Özcan, E. (2013). A two stage approach for high school timetabling. In International Conference on Neural Information Processing (pp. 66-73). Springer Berlin Heidelberg.)

Shambour, M. K. A. T., Khader, A. A., \& Shambour, Q. (2014). Modified tournament harmony search for unconstrained optimisation problems. In Recent Advances on Soft Computing and Data Mining (pp. 283-292). Springer, Cham.

Shambour, M., K. (2017). Dynamic search zones for harmony search algorithm. In Proceedings of 8th International Conference on Information Technology, Jordan, 8, 941-946. doi: 10.1109/ICITECH.2017.8079972. Suganthan, P. N., Hansen, N., Liang, J. J., Deb, K., Chen, Y. P., Auger, A., \& Tiwari, S. (2005). Problem definitions and evaluation criteria for the CEC 2005 special session on real-parameter optimization (Technical report No. 200505). Retrieved from http://www.ntu.edu.sg/home/ EPNSugan.

Sultan, A. B. M., Sulaiman, R. M. M., \& Bakar, M. R. (2004). A genetic algorithm approach for timetabling problem: The time group strategy. Journal of Information and Communication Technology (JICT), 3(2), $1-14$.

Valipour, K., \& Ghasemi, A. (2017). Using a new modified harmony search algorithm to solve multi-objective reactive power dispatch in deterministic and stochastic models. Journal of AI and Data Mining, 5(1), 89-100.

Wang, G. G., Gandomi, A. H., Zhao, X., \& Chu, H. C. (2016). Hybridizing harmony search algorithm with cuckoo search for global numerical optimization. Soft Computing, 20(1), 273-285.

Xu, H., Gao, X. Z., Wang, T., \&Xue, K. (2010). Harmony search optimization algorithm: application to a reconfigurable mobile robot prototype. In Recent Advances in Harmony Search Algorithm (pp. 11-22). Springer Berlin Heidelberg.

Yassen, E. T., Ayob, M., Nazri, M. Z. A., \& Sabar, N. R. (2015). Metaharmony search algorithm for the vehicle routing problem with time windows. Information Sciences, 325, 140-158.

Yong, Z., \& Sannomiya, N. (2000). A method for solving large-scale flowshop problems by reducing search space of genetic algorithms. 2000 IEEE International Conference on Systems, Man, and Cybernetics, 3, 17761781.

Zeng, B., \& Dong, Y. (2016). An improved harmony search based energyefficient routing algorithm for wireless sensor networks. Applied Soft Computing, 41, 135-147. 
Zhang, B., Pan, Q. K., Zhang, X. L., \& Duan, P. Y.(2015). An effective hybrid harmony search-based algorithm for solving multidimensional knapsack problems. Applied Soft Computing, 29,288-297. 